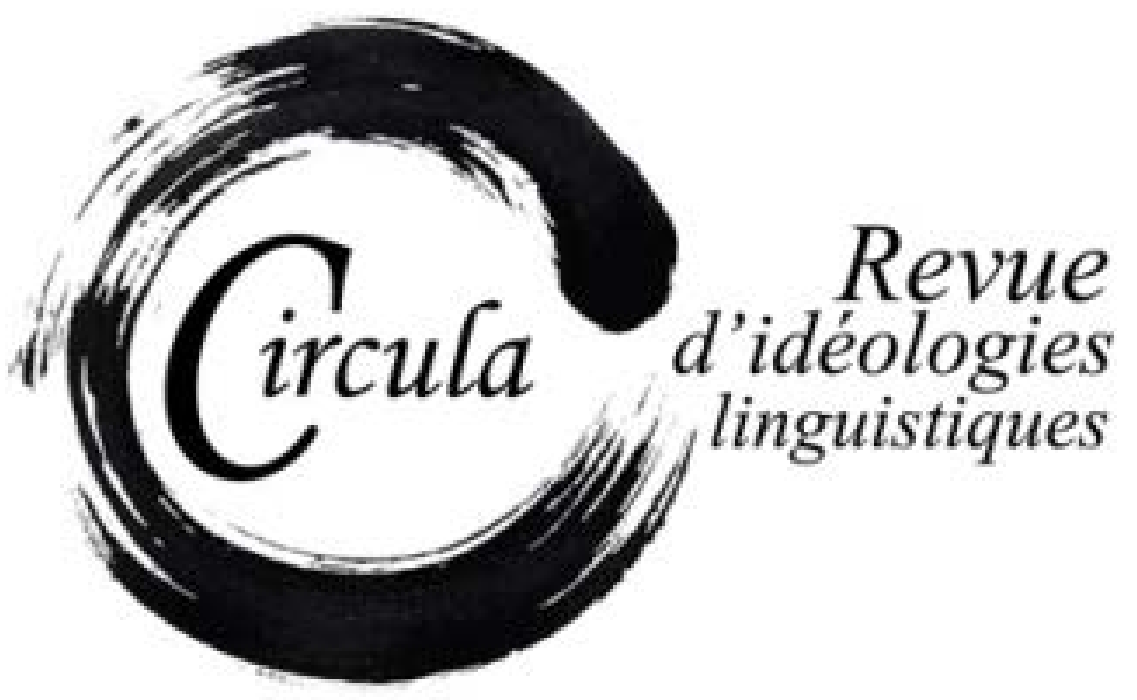

TITRE: LA SEMPLICITÀ E LA SEMPLIFICAZIONE: FRA L'IDEOLOGIA E LA RETORICA AUTEUR(S): MARIA ZAŁĘSKA, UNIVERSITÀ DI VARSAVIA

Revue: CIRCULA, NUMÉRO 2, PAGES 192-211

ISSN: 2369-6761

Directeurs: Wim Remysen et SABINE SCHWARZE

URI: HTTP://HDL.HANDLE.NET/11143/7990

DOI: $10.17118 / 11143 / 7990$ 


\section{La semplicità e la semplificazione: fra l'ideologia e la retorica}

Maria Załęska, Università di Varsavia maria.m.zaleska@gmail.com

Riassunto: La ricerca analizza i contributi di un'accesa disputa mediatica risalente al 2011. La disputa è stata scatenata dalla notizia che il noto libro /l nome della rosa di Umberto Eco sarebbe stato semplificato. La categorizzazione dei contributi proposta in questa ricerca si ispira al modello retorico dello status quaestionis. L'analisi permette di rilevare che la controversia sulla semplificazione e sulla semplicità riguardava in particolare lo status qualitatis, e, in minor misura, lo status definitionis. Lo studio ricostruisce due ideologie contrastanti della semplicità e della semplificazione che sottostanno agli argomenti usati nella disputa. È a causa della sovrapposizione delle due nozioni che la controversia si è rivelata tanto accesa.

Parole chiave: semplicità; semplificazione; disputa mediatica; retorica; status quaestionis

Abstract: The paper analyzes a series of contributions to a mediatic dispute that took place in the year 2011 and was initiated by the news that the famous book The name of the rose by Umberto Eco was allegedly simplified. The categorization of the contributions proposed in the paper is inspired by the rhetorical model of status quaestionis. The analysis permits to establish that the controversy about simplification and simplicity has been formulated mainly within the status qualitatis and, to a lesser degree, within the status definitionis. The study reconstructs two contrasting ideologies of simplicity and simplification underlying the arguments used within the dispute. The controversy turned out to be very animated because the two notions have been blurred.

Keywords: simplicity; simplification; mediatic dispute; rhetoric; status quaestionis 


\section{Introduzione}

«Bisogna scrivere nel modo più semplice possibile - ma non ancor più semplice»: questa frase, attribuita ad Einstein, introduce nel vivo di un caso mediatico internazionale, risalente al 2011. La disputa, condotta sui giornali e su Internet, riguardava la semplificazione de Il nome della rosa di Umberto Eco. La discussione mediatica, svoltasi prima dell'uscita nelle librerie della versione semplificata, quando i partecipanti non conoscevano la reale portata delle modifiche, si basava sulle ideologie ricorrenti relative alla semplificazione e al concetto cui essa rimanda - la semplicità.

La controversia riguardava tre problemi interrelati, concernenti i modi di trattare un capolavoro: (a) Modificare o no?; (b) Se modificare - in che modo modificare: semplificando? Complessificando? Scrivendo una versione alternativa?; (c) Se modificare semplificando: come concretamente si dovrebbe (o potrebbe) manifestare questa semplificazione?

La ricerca si prefigge tre obiettivi: descrittivo, analitico e interpretativo. A livello descrittivo, lo scopo è quello di presentare i contributi autentici alla disputa che - tramite la loro animosità, ironia, emozionalità - svelano l'importanza dei concetti di semplicità e di semplificazione. Per analizzarli, è stato adottato uno strumento retorico - il modello dello status quaestionis - utile per categorizzare le funzioni degli enunciati espressi nella disputa. L'analisi permette di rilevare l'esistenza di due ideologie della semplicità e della semplificazione. Ė proprio il mancato riconoscimento della differenza fra di loro che ha alimentato discussioni così accese.

\section{Corpus della ricerca}

Nel luglio 2011 sul sito dell'editore Bompiani è apparso un annuncio promozionale sull'imminente uscita in libreria, prevista per il 5 ottobre dello stesso anno, de Il nome della rosa nella versione «più accessibile ai nuovi lettori». Questa «accessibilità» era trattata come una caratteristica tanto positiva da usarla come esca nella promozione del libro. Anche Umberto Eco in un'intervista concessa nell'agosto 2011 al Corriere della Sera preannunciava la semplificazione della propria opera.

La notizia è stata diffusa in diversi media italiani e stranieri. Ė stato un critico letterario francese, Pierre Assouline, a scatenare la disputa mediatica con il suo articolo pubblicato su Le Monde, dal titolo eloquente «Eco réinvente son "Nom de la rose" pour les nuls». La disputa - sulla carta stampata e in Internet - ha coinvolto giornalisti, critici della letteratura e lettori. Alcuni si schieravano contro la semplificazione, altri invece condividevano essenzialmente l'idea della semplificazione presentata dell'autore.

Già, l'idea: la disputa mediatica si svolgeva prima che i disputanti avessero la possibilità di consultare il libro. Di conseguenza, tutto ciò che è stato detto non si poteva basare sui fatti, bensì sulle idee ed ideologie (per la definizione, v. sotto). Tali ideologie saranno ricostruite in base a un corpus com- 
posto di reazioni mediatiche alla notizia presentata sul sito Bompiani. La reale portata della disputa nel 2011 era molto più accesa, rispetto a quello che nel 2013 - l'anno in cui è svolta la ricerca - era ancora accessibile su Internet. Infatti, come risulta da alcuni accenni negli articoli e/o dai comunicati pubblicati sotto gli articoli consultabili nella versione elettronica, dopo l'apice della disputa, molti dei commenti sono stati soppressi e non sono più disponibili all'ispezione.

Per tutti i testi del corpus elencati sotto, la data d'accesso è il 10 settembre 2013. I titoli e i contenuti sono riportati nella grafia originale, con i refusi e gli errori. Il corpus è composto di tre categorie di testi. La prima è formata dagli articoli degli specialisti nel senso lato del termine (i giornalisti, gli studiosi di letteratura, gli autori dei blog specializzati, v. sotto, Fonti primarie, la prima categoria dei testi).

La seconda categoria la costituiscono i contributi dei «lettori comuni»: i commenti sui siti web dei giornali, le prese di posizione sui blog e sui forum di discussione, le notizie anonime dai giornali elettronici (v. sotto, Fonti primarie, la seconda categoria dei testi).

Infine, come una categoria a parte, viene acclusa un'intervista fatta da Paolo Di Stefano con Umberto Eco, pubblicata già dopo l'uscita in libreria de /l nome della rosa in versione semplificata, v. sotto, Fonti primarie, la terza categoria). Sotto l'articolo nella versione elettronica appare il comunicato: «i commenti sono stati disabilitati», dunque non sono più consultabili.

\section{Quadro teorico-metodologico}

La semplicità e la semplificazione (nonché le loro controparti: la complessità e la complessificazione) risultano concetti teorici molto difficili, esplorati dalla prospettiva linguistica, testuale e ideologica. L'etimologia del termine semplice, da cui derivano i due sostantivi sopraelencati, è illuminante:

sémplice 1 (ant. simplice) agg. [lat. simplex simplǐcis, comp. della radice *sem- «uno, uno solo» (cf. semel) e di una radice * plek-presente in plectěre «allacciare», plicare «piegare» (cf. duplice, triplice, ... molteplice)]. - 1. Che è costituito di un solo elemento e non può risolversi perciò in ulteriori componenti. ${ }^{1}$

A livello linguistico, si studiano i meccanismi della semplificazione sintattica e semantica (Voghera, 2001; Fiorentino, 2009), nonché i meccanismi coinvolti nella variazione diamesica tra la scrittura e l'oralità (Voghera, 1982; Baldini, 1989).

Nei testi si cercano i correlati linguistici della semplicità (Piemontese, 1996). Le dinamiche complesse fra la semplicità e la complessità come proprietà di ragionamento nei testi letterari, scientifici nonché nell'arte sono esplorate in Merrell (2001). La (troppa) complessificazione è studiata in riferimento ai testi burocratici (Fioritto, 1997) e ai testi scientifici (Załęska, 2008, 2009). Il concetto di

1. http://www.treccani.it/vocabolario/tag/semplice/ 
semplicità riferito ai testi, nonché le pratiche di semplificazione, vengono connotati positivamente. Di fronte a questa connotazione essenzialmente positiva dei concetti di semplicità e semplificazione, è interessante esplorare il perché della controversia sulla semplificazione de ll nome della rosa.

Una tale controversia sembra essere dovuta a idee ben diverse su cosa sia la semplicità e la semplificazione. Idee - o ideologie? Nei testi sull'ideologia si sottolinea l'inerente vaghezza del concetto, nonché le svariate connotazioni del termine (Ponzio, 2006a, 2006b; van Dijk, 1998). Nell'accezione comune, l'ideologia è definita come un sistema di idee per definizione oppressivo, imposto e falso, dunque connotata negativamente. L'ideologia è usata qui in un'accezione più larga, così come l'intende van Dijk (1998). L'ideologia è trattata come un sistema di idee che costituisce una forza a volte oppressiva, a volte «consensuale e benefica» (van Dijk, 1998: 163). Nel modello di van Dijk (1998) le ideologie non sono circoscritte alle relazioni sociali della dominazione, bensì permeano qualsiasi relazione: vengono attuate (ingl. performed) nelle interazioni quotidiane e tramite le interazioni quotidiane. Nel suo modello, ciò che conta non è il valore della verità (ingl. truth value) dell'ideologia, bensì gli effetti di verità (ingl. truth effects) che hanno un ruolo cognitivo e sociale nella gestione del pensiero e dell'interazione (van Dijk, 1998: 315). Negli approcci moderni, il concetto di ideologia è esplorato dalla prospettiva del discorso che la crea, esprime e riproduce (van Dijk, 1998: 5). Se da una parte questo è un vantaggio, dall'altra, però, rischia di diluire il concetto di ideologia con i concetti alternativi, quali stereotipi, pregiudizi o l'immagine linguistica del mondo.

In quanto segue, i modi di esprimersi nella summenzionata disputa a proposito della semplicità e della semplificazione saranno trattati come costitutivi delle rispettive ideologie, attuate e ripetute tramite il discorso (l'uso dei sostantivi di un particolare campo semantico; la loro valutazione; le affermazioni a proposito, gli argomenti pro e contro).

Come una griglia analitica per i contributi reperibili in rete, è stato adottato il modello retorico dello status quaestionis, fatto risalire a Ermagora ed Ermogene (v. Patillon, 1988; Heath, 1994; v. anche il testo originale di Ermogene, la traduzione a cura di Podbielski, 2012). Originariamente, il modello serviva per controllare la dinamica della disputa nel contesto giudiziario, quando l'accusatore e il difensore lottavano per l'impostazione della causa conforme ai loro ruoli istituzionali: per o contro l'accusato. In questa situazione prototipica, si trattava di un'interazione concreta fra due persone concrete a proposito di un fatto avvenuto.

Nel caso della disputa mediatica in questione, la situazione comunicativa è diversa: i partecipanti sono tanti, differiscono per il grado di preparazione professionale; la loro interazione non ha una struttura chiara, visto che sempre nuove persone si uniscono alla corrente dei commenti pro e contro. Di conseguenza, il modello di status quaestionis sarà adattato non tanto per ricostruire la dinamica effettiva della disputa nella sua cronologia, bensì nella tipologia dei suoi contributi. 


\section{Status quaestionis nella ricostruzione della disputa}

Nella griglia analitica adottata sotto, ciascuno dei quattro status quaestionis raggruppa i contributi degli specialisti, dei lettori e dell'autore stesso, mostrando le differenze dovute alle ideologie della semplicità adottate.

\subsection{Fatti (status coniecturalis)}

Nel modello retorico originale, la prima tappa di ogni disputa - lo status coniecturalis - riguarda i fatti. Nell'ambito del discorso giuridico, che è servito come modello per elaborare il quadro teorico dello status quaestionis, si tratta di stabilire che cosa effettivamente è successo: Xè morto. Accanto a lui si trova Y. Sono i fatti interrelati o meno? Chi è responsabile? Le domande specifiche riguardano dunque i fatti (Che cosa è successo?) ed, eventualmente, la responsabilità per i fatti (Chi ha fatto che cosa? A chi?).

Nella disputa su Il nome della rosa, ai partecipanti non erano noti i fatti stessi: il libro nella versione semplificata all'epoca non era ancora uscito in libreria. Il sito Bompiani informava, alquanto genericamente, che erano stati introdotti cambiamenti. Tali informazioni generiche sono state diffuse dai giornalisti:

(1) Il best-seller internazionale che ha venduto 30 milioni di copie al mondo, che è stato tradotto in più di 40 lingue, si presenterà ad ottobre con un nuovissimo look, con un linguaggio più fresh e passaggi più flash.

(2) Il progetto di revisione voluto dal semiologo di Alessandria non ha intaccato la trama, ma ha riguardato la scrittura, ripensata per coinvolgere un pubblico di «nuovi lettori».

Nulla però è cambiato in struttura, trama e intrighi.

Mentre alcuni formulavano le asserzioni, altri preferivano modalizzare l'informazione:

(3) [...] una versione del testo «più accessibile ai nuovi lettori», il che va tradotto probabilmente in una semplificazione del linguaggio e dei passaggi più ardui della trama.

I dettagli di questo cambiamento risultavano ignoti. I partecipanti alla disputa dovevano dunque ammettere la propria incertezza a proposito dei fatti, dichiarando di poter soltanto formulare supposizioni a proposito delle modifiche introdotte:

(4) On ne sait pas encore précisément comment il va s'y prendre mais on sait qu'il l'a revisité et réinventé en ce sens. 
(5) Todavía no se conocen los detalles de la reinvención del gran clásico de la literatura italiana y mundial.

I non-specialisti si permettevano supposizioni molto più ardue, più fantasiose e più ironiche, divertendosi a immaginare le possibili modalità di semplificazione. Dai loro contributi risulta che si immaginavano la semplificazione in termini stilistici (la semplificazione come ricorso alle soluzioni stilistiche preferite dai giovani) e in termini cognitivi (la semplificazione come aggiornamento della trama alle circostanze moderne). Tali idee sulla semplificazione traspaiono sia dai giochi linguistici con il titolo dell'opera (l'elemento che, com'era risaputo da tutti, non è stato cambiato) e dalle trovate a proposito della trama.

Nel primo caso, l'idea della semplificazione era resa tramite la riduzione formale, ovvero l'eliminazione delle parole dalla forma completa (Il nome della rosa) a una parola sola, addirittura abbreviata come vezzeggiativo:

(6) Rosa

(7) Rò (per gli amici)

La semplicità associata con la brevità formale si manifesta anche tramite l'allusione agli standard della brevità, caratteristici per la scrittura elettronica:

(8) Il nm dla rs.

(9)IInome della Ros@.

(10) Ce 1 frate ke scp!

Una tecnica opposta alla riduzione formale è la ripetizione, secondo le modalità caratteristiche per il linguaggio orale:

(11) Rosa, rosa, rosa

(12) Eco semplifica «ll Nome della Rosa»: osa... osa... osa...

Un altro modo di rendere l'idea della semplificazione è l'aggiornamento della trama alle circostanze o agli schemi moderni, facilmente riconoscibili ai giovani lettori:

(13) Il libro proibito sarà la grammatica.

(14) La ragazza dopo averla data al frate diventerà madre superiora.

(15) Invece che al rogo gli eretici vengono condannati a un ban definitivo. 
(16) Guglielmo e Adso sono 2 smanettoni. Fanno un casino con Wiki xciò va riformattato tutto, ma il pc va a fuoco e allora tutti in discoteca (fine).

Un altro modo di suggerire l'idea della semplificazione è l'infantilizzazione:

(17) In omaggio con il libro i pennarelli per colorare le figure.

(18) Sarà una confezione-gioco di Gormiti vestiti da frati.

Anche il cambiamento del genere del discorso rendeva l'idea della semplificazione:

(19) Diventerà un fotoromanzo!

(20) «[...] se proprio dovevano tirar fuori un'iniziativa semplificata» e chiaramente commerciale, potevano pensare a una versione a fumetti!

A differenza dei giornalisti, critici della letteratura o del pubblico, coinvolti tutti nelle supposizioni sui fatti, l'autore del libro conosceva perfettamente le modifiche introdotte. Per identificare i fatti, in uno degli articoli si citavano le parole dell'autore:

(21) La nuova edizione del romanzo è comunque in arrivo e «la bagarre» sulla presunta riscrittura, nasce dalla comunicazione in merito della casa editrice che parlava dell'impegno di Eco «per renderlo più accessibile ai nuovi lettori». Per lo scrittore è un'espressione curiosa che forse voleva suggerire ai librai che una nuova edizione avrebbe attirato l'attenzione di nuovi lettori [...], da leggere comunque alla luce dell'esplicito passaggio che recitava: «Non lo ha riscritto come hanno fatto altri autori». «Ci sarà scritto nel colophon 'edizione riveduta e corretta', come accade per molti libri quando dopo tanti anni si fa una seconda edizione. Sono intervenuto anzitutto - spiega Eco - su alcune inesattezze, a eliminare ripetizioni di uno steso termine e poche pagine di distanza, spesso mi sono preoccupato del ritmo, perche' basta rimuovere un aggettivo o togliere un inciso per rendere piu' aereo un intero periodo». «Forse l'unica variazione di sostanza e' nella descrizione del bibliotecario, perchè volevo togliere un fastidioso riferimento neogotico. Cosa di poche righe», assicura Eco.

Nell'intervista del gennaio 2012, dunque posteriore sia alla disputa che all'uscita del libro nelle librerie, Umberto Eco in quanto autore ha reagito a quello che gli è stato imputato dai partecipanti alla disputa. Ha evidenziato tra l'altro anche i cambiamenti nella costruzione dei personaggi:

(22) Il fatto è che quando scrivevo I/ nome della rosa mi compiacevo di un certo gusto citazionistico, poi diventato il segno del postmoderno, che non era ancora così diffuso. Dopo trent’anni uno dice: diamoci una regolata... Nel ritratto del bibliotecario c'era una citazione dal Confessionale dei penitenti neri. A distanza di tempo non mi sembrava indispensabile e 
I'ho tolta. A Guglielmo ho tagliato i ciuffi di peli giallastri alle orecchie, ma non c'entra il fatto che volevo farlo somigliare a Sean Connery, come è stato detto. (Di Stefano, 2012)

Le affermazioni dell'autore mostrano la distanza tra i fatti reali e le supposizioni o addirittura le fantasticherie del pubblico giovanile a proposito della semplificazione.

\subsection{Definizioni (status definitionis)}

Nel modello retorico della disputa, la seconda tappa, chiamata status definitionis, riguardava la definizione dei fatti. Il punto di riferimento originale era la disputa in tribunale: se i fatti si sono rivelati indisputabili (per es. qualcuno è morto), allora si poteva accusare o difendere, rendendo disputabile la definizione del fatto: si tratta di una morte naturale o piuttosto di un omicidio? Come si evince da questo esempio, nello status definitionis non ci si riferiva alla definizione nel senso stretto del termine (il genus proximum e la differentia specifica), bensì della scelta dei termini con cui nominare i fatti. Dal punto di vista linguistico, vengono usati in primo luogo i sostantivi (per es. la semplificazione del testo), i verbi (semplificare il testo), ma vi possono apparire anche gli aggettivi (per es. il testo nella versione semplificata).

Se è nota soltanto la notizia del fatto, e non il fatto stesso, è difficile distinguere lo status coniecturalis dallo status definitionis, ossia distinguere i fatti dai nomi con cui essi vengono identificati nei testi che li riportano. Il fatto del cambiamento rispetto alla versione precedente de Il nome della rosa nella discussione mediatica è stato identificato con vari nomi. Alcuni termini sono generici e accennano alla novità e all'attualizzazione (reinvenzione, riscrittura, revisione; la versione rifatta, rivisitata, riveduta, aggiornata; un «ritocchino»; il linguaggio rinfrescato; pretende actualizar su novela).

Altri termini sono invece più precisi, specificando anche il tipo del cambiamento: non è solo la categoria della novità, ma anche quella della facilità (la semplificazione, la versione più accessibile, il testo senza spine; facilitare il linguaggio; renderlo più «digeribile»):

(23) Eco semplifica «ll Nome della Rosa» per le nuove generazioni.

(24) Eco senza spine. «ll Nome della Rosa» in versione semplice per i più giovani.

(25) «ll nome della rosa» nella versione «più accessibile ai nuovi lettori».

(26) [...] una nueva versión más «ágil» [...].

La «definizione» delle azioni costitutive per la semplificazione rimane comunque a livello alquanto generico: 
(27) Verranno infatti snelliti alcuni passi, forse alcune citazioni latine e digressioni filosofiche e religiose, probabilmente le famose prime cento pagine lente ed «imposte» scorreranno con più ritmo, mentre la trama e gli intrighi resteranno invariati.

Nella «definizione» dei nomi di queste azioni spesso viene sfruttata l'ambiguità delle virgolette: da una parte esse indicano la citazione della versione originale, dal sito Bompiani, dall'altra segnalano una presa di distanza nei confronti dell'informazione solo riportata, non acquisita di prima mano:

(28) Pour le rendre "plus accessible» aux nouveaux lecteurs, notamment ceux qui ne connaissent le roman que par les sites internet, «faciliter» certains passages et «rafraîchir» la langue $[\ldots]$.

Nella suddetta intervista con Di Stefano, Umberto Eco si è opposto ai tentativi di «definizione» che sono apparsi nella disputa mediatica:

(29) Su Internet continuano a parlare della «riscrittura» del Nome della rosa, anche se non è una riscrittura, per cui se alla fine ci saranno degli ingenui che la comperano credendola diversa, peggio per loro. Sono stati avvertiti in tutti i modi.

Altre «definizioni» delle azioni coinvolte nella semplificazione appariranno negli esempi sotto.

\subsection{Qualifiche e valutazioni (status qualitatis)}

Nel modello retorico relativo alla dinamica della disputa, la terza tappa, chiamata status qualitatis, riguarda la qualifica dei fatti, già definiti tramite la nomenclatura. Se è indisputabile che si tratta di un omicidio, l'avvocato, volendo difendere il cliente (o l'accusatore, intento a condannare l'imputato) può rendere disputabile la qualifica dell'atto: era un omicidio colposo o forse casuale?

Dal punto di vista linguistico, nello status qualitatis le qualifiche si esprimono prevalentemente con gli aggettivi, ma anche con i sostantivi o i verbi che veicolano una connotazione. Nella disputa sotto, alle manifestazioni di questa categoria si ascrivono anche le valutazioni, gli atti espressivi e le argomentazioni (le opinioni e gli argomenti a supporto).

Nella discussione mediatica discussa sopra, dopo aver identificato che si tratta della semplificazione, si procede alla sua qualifica: è una semplificazione «colposa», valutata negativamente, oppure una semplificazione «meritevole», valutata positivamente?

La maggior parte dei partecipanti alla disputa ha qualificato il fatto del cambiamento, definito come semplificazione, come «colposa», sottoponendola a forti critiche. In primo luogo, i sostenitori di quest'opinione partivano dal presupposto che un'opera letteraria, già pubblicata, non va più 
cambiata, tanto più un'opera reputata come perfetta (v. l'etimologia del termine perfetto ${ }^{2}$, v. anche lo studio di Tatarkiewicz, 1976 sulla perfezione).

Di conseguenza, ogni modifica all'interno di un'opera letteraria ritenuta (ragionevolmente) perfetta passa per una manomissione.

(30) Sacrilegio. Il nome della Rosa è perfetto così com'è. Tra l'altro lo trovo un libro veramente divertente, per tutte le citazioni nascoste e i giochi dirimando, che sono una vera gioia a ogni rilettura.

(31) Sono vivamente contraria e credo che in questo modo la letteratura, soprattutto quella alta, ceda il passo al marketing editoriale. Non ci sto!

In secondo luogo, fra diverse possibili forme di cambiamento, quella che consiste nella semplificazione risulterebbe particolarmente condannabile. Da questa prospettiva, la semplificazione significa infatti danneggiare l'originale, togliendogli ciò che è costitutivo per la sua qualità, che lo rende riconoscibile e unico:

(32) Ammetto di essere stata sconcertata da tale dichiarazione e dal progetto di rinnegare la propria opera.

Viene dunque criticata la semplificazione sotto forma di aggiornamento, percepita come una contraffazione delle circostanze storiche:

(33) [...] in realtà non fa altro che snaturare un'opera il cui valore intrinseco dipende anche dall'essere prodotto [...] di un'epoca culturale, di un linguaggio, di una temperie.

Similmente, è condannata anche la semplificazione lessicale e sintattica che toglie la bellezza all'originale:

(34) [...] semplificare il linguaggio, per renderlo accessibile ai giovani di oggi, mi sembra non

abbia senso, in quanto la peculiarità de «ll nome della rosa» è il suo particolare linguaggio.

Dalle formulazioni sopraccitate si evince la connotazione negativa del concetto di semplificazione, associato con la distruzione, la manomissione e lo snaturamento.

In terzo luogo - se la semplificazione in sé è un intervento condannabile, lo è ancor di più se viene interpretata nella luce del rapporto autore/pubblico. La semplificazione, infatti, sarebbe introdotta

2. «Perfètto = lat. PERFÈCTUS da PERFÍCERE finire, compire, composto della part[icella] PER che nella composizione dei verbi aggiunge loro l'idea di compimento (v. Per e FÀCTUS (nei composti FĖCTUS) part. pass. del verbo FÀCERE fare [...]). Che non ha alcun mancamento, Intiero, Compiuto, Eccellente, alla pari dell'essere supremo che non ha difetti» (http:// www.etimo.it/?term=perfetto; data d'accesso il 12 settembre 2013). Secondo Aristotele (Metafisica, libro Delta, V, 16, 1021 b 12 n), perfetto è ciò che contiene tutte le parti necessarie. 
dall'autore in funzione di come s'immagina il pubblico. Nella disputa mediatica, il pubblico a cui l'autore si riferirebbe è stato «qualificato» in vari modi, da quelli neutrali a quelli palesemente valutativi. Appaiono infatti le categorie generiche come la novità (per le nuove generazioni...; ai nuovi lettori...) e l'età (per i giovani; per i più giovani). Nel contesto della semplificazione, sono interessanti le categorie specifiche relative alla preparazione intellettuale del pubblico: si tratta non solo delle competenze dominanti (per i nativi digitali), ma anche delle incompetenze dominanti (per una generazione culturalmente inferiore; un pubblico pigro), fino a squalificare i lettori (pour les nuls...; for dummies...; quest'ultimo è un'allusione a una serie popolare di manualetti divulgativi destinati proprio for dummies). Al pubblico nuovo, giovane, competente nell'ambito digitale e incompetente nell'ambito culturale viene contrapposto, seppur implicitamente, un pubblico dei tempi passati, non più giovane, forse incompetente nell'ambito digitale, ma ritenuto competente nell'ambito culturale. Mentre quest'ultimo era preparato a fruire delle opere complesse della cultura, il pubblico nuovo sarebbe bisognoso di semplificazione a causa delle proprie gravi lacune:

(35) El objetivo es que quienes solo conocen la obra por citas de Internet, asustados por su dificultad idiomática o por lo denso de algunos pasajes, puedan leerla también, así como los lectores digitales.

Dal punto di vista retorico, l'adattarsi al pubblico è una manifestazione dell'aptum. È una categoria retorica, individuata all'interno dell'elocutio retorica (cioè la tappa della creazione metodica del testo che consiste nella scelta della veste linguistica in cui sarà presentato il contenuto). Realizzare aptum significa che l'autore prende in considerazione le aspettative del pubblico, fornendo il messaggio nella forma che sia comprensibile e attraente per il pubblico in questione.

Ma come si autopercepiva il pubblico? Alcuni lettori hanno ammesso esplicitamente la propria inferiorità culturale, aspettando fiduciosi la versione semplificata:

(36) Finalmente questo libro sarà accessibile anche per me. Non vedo l’ora.

Altri mettevano in dubbio l'utilità di tutta l'operazione:

(37) Secondo me i nativi digitali al massimo lo useranno come base per il monitor.

(38) Eco semplifica Il Nome della Rosa per le nuove generazioni. Ora le vecchie potranno chiedere spiegazione a loro. (No, sai com'è... Non c'ho mai capito un cazzo[.])

Qualche lettore contestava l'associazione fra la giovinezza e l'incompetenza culturale:

(39) lo lo lessi con profondo entusiasmo in terza media, non incontrando ostacoli o difficoltà di sorta. 
Anzi, in altri commenti si sottolineava che il fattore di età è irrilevante: è proprio la complessità originale dell'opera che fa individuare i lettori all'altezza:

(40) [...] non capisco il senso di eliminare le «trappole» che Eco mise nel primo, in cui le prime [...] 100 pgg sono messe lì proprio per fare una selezione dei lettori.

Anzi, l'idea che l'opera dovrebbe essere accessibile proprio a tutti, a prescindere dalla rispettiva preparazione culturale di ciascuno, viene ridicolizzata e rifiutata:

(41) Eco semplifica il romanzo /l nome della rosa, per renderlo agevole a ogni lettore. Inorridiamo al solo pensiero e ne ridiamo con Gramellini sulla Stampa di sabato 20 agosto.

Anche gli specialisti - i critici della letteratura o i giornalisti - contestavano l'associazione, imputata all'autore, tra la giovinezza e l'ignoranza, che l'avrebbe portato a creare una versione semplificata:

(42) Eco se serait-il cyniquement convaincu que les jeunes générations du début du XXlème siècle largement numérisées sont déjà moins cultivées que les précédentes au point de leur proposer un Nom de la rose pour les nuls avec qu'il [sic] faut de liens hypertexte pour pallier leur ignorance crasse?

(43) [...] l'operazione è apparsa come un tentativo di venire incontro a una generazione culturalmente inferiore, che a differenza di quella precedente, cresciuta prima e durante gli anni Ottanta, ha smarrito la capacità di comprendere i paragrafi in latino, le dispute tra domenicani e francescani e i riferimenti aristotelici.

Di conseguenza, la versione semplificata, che sarebbe ideata per gli ignoranti, era ritenuta indegna della gente di un certo livello culturale. L'ironia è molto eloquente:

(44) Una versione user-friendly?

(45) Come si intitolerà «ll nome della rosa versione for dummies»?

(46) Le roman était formidable, mais non, pitié, pas le Nom de la rose le retour, pas la saison 2!

Anziché l'applicazione dell'aptum retorico, la semplificazione era dunque interpretata come il disprezzo per il pubblico reputato ignorante:

(47) Et en passant, quel mépris pour la nouvelle génération de lecteurs!

(48) Nel corso del 2012 usciranno le traduzioni straniere della versione facilitata. Bene, allora Eco è convinto che tutto il mondo si stia rimbecillendo, non solo l'Italia. [...] stavolta ha allargato gli orizzonti. 
Nella disputa sono apparsi i commenti irritati del pubblico che ha trattato la semplificazione come un'offesa:

(49) [...] non è mancata la reazione offesa del pubblico, sdegnato per questo indesiderato aiuto arrivato dallo scrittore.

(50) [...] quando è cominciata a circolare la notizia di questo intento di semplificazione operato dal semiologo, un po' ovunque i lettori hanno incominciato a storcere il naso, soprattutto i giovani che avrebbero dovuto beneficiare di questo accorgimento stilistico, quasi a dover ammettere tacitamente la propria incapacità di lettura.

(51) Ohibò, io ho letto «ll nome della rosa» a sedici anni [...]. Vuoi che i sedicenni di adesso non riescano a capire 'sto testo? Saranno mica diventati più stupidi?

Dai commenti presentati sopra risulta che, agli occhi del pubblico, niente poteva giustificare la semplificazione, interpretata come un abbassamento del livello.

(52) [...] boh, anche io non mi spiego questa caduta di stile a 80 anni suonati.

(53) Eco semplifica Il Nome della Rosa per le nuove generazioni. Questa si che si chiama speranza per il futuro.

La semplificazione era contestata anche per motivi etici. Le accuse coinvolgevano l'etica dell'arte, in quanto un letterato non dovrebbe danneggiare un capolavoro per compiacere gli ignoranti. Veniva pure affrontata l'etica della didattica: un professore universitario, insegnando in cosa consista la bellezza della letteratura, non avrebbe mai dovuto contribuire lui stesso a distruggere un capolavoro. L'aggravante era anche l'imputazione che il testo è stato semplificato per motivi di lucro, allo scopo di attirare più clienti con la merce intellettualmente a buon mercato:

(54) In questi giorni dovrebbe uscire una versione «semplificata» de II nome della rosa. [...] Personalmente l'idea mi ripugna e la trovo soltanto una mossa commerciale piuttosto squallida $[\ldots]$.

(55) Tuttavia sono del parere [...] che sia solo una studiata editoriale per venderne altre ulteriori copie (io a casa ne ho tre edizioni per esempio e sarò costretto, mi sa, a procurarmi pure quest'altra!)

(56) On voit ce que la librairie, l'édition et l'auteur peuvent y gagner, mais la littérature? On aimerait après cela écouter le professore Eco expliquer à ses étudiants les nécessités de l'écriture, l'économie interne d'une $[$ sic] récit, les exigences d'un texte. [...]

Riassumendo, la semplificazione, intesa come un abbassamento di livello, faceva mettere in dubbio la credibilità dell'autore in quanto intellettuale e in quanto cultore della lingua: 
(57) Chi lo avrebbe mai detto che i linguisti sono così poco puristi?

I sostenitori di questa visione della semplificazione riaffermavano invece il valore della versione originale che non dovrebbe essere mai toccata:

(58) lo ho reagito [...] con la ri-lettura dello splendido originale.

(59) [...] ci scommetto, la versione de «ll nome della rosa» che appassionerà i lettori fra cent’anni non sarà quella riveduta e corretta, [...] ma quella originale, tortuosa, complessa e bellissima del 1980.

Molto meno numerosi erano i giudizi positivi sulla semplificazione preannunciata. In essi, in primo luogo, alla domanda: è lecito modificare un'opera letteraria o no? - si dava una risposta affermativa. Anche se è un capolavoro di letteratura, può pur sempre essere imperfetto - dunque rimane potenzialmente perfezionabile dal suo autore:

(60) [...] un autore ha il sacrosanto diritto di cambiare e rieditare quello che scrive (in particolare se l'autore è un genio come Eco).

(61) D’altronde ogni autore ha il diritto e il dovere di tornare sulle proprie opere e finché è possibile perfezionarle, così come ogni lettore ha il diritto e il dovere di decidere se leggere o meno, sia che l'opera in questione sia particolarmente «difficile» o semplicemente noiosa.

Come ricorda Di Stefano nel suo articolo dedicato al caso de Il nome della rosa racchiuso nel corpus, la riscrittura era una pratica comune fra i letterati e poeti. Nella letteratura italiana, basti accennare ai nomi di Ariosto, Gadda o Manzoni che - per la propria sete di perfezione (e non per adattarsi a un pubblico ignorante) - continuavano a modificare le rispettive opere. Anzi, è nato addirittura tutto un filone di ricerche dedicato alle riscritture delle opere letterarie (Raboni e Italia, 2011).

In secondo luogo, a detta dei fautori di questa posizione, fra diverse possibilità di modificare un'opera letteraria, la modifica che consiste nella semplificazione è particolarmente apprezzata. La semplificazione è infatti presentata come un processo di perfezionamento dall'ancora troppo complicato verso una semplicità ideale (o, meglio, verso la semplessità, il miscuglio particolare della semplicità e della complessità, v. Berthoz, 2011). La semplificazione viene vista come il processo di eliminazione di tutto quanto è inutile, il che porta a una costruzione economica del messaggio. La semplificazione viene trattata come un lavoro sull'equilibrio precario tra la forma e il contenuto: la forma estetica del messaggio (lo stile, il ritmo, la melodia, la struttura dell'opera) ha gli effetti immediati sulla percezione del contenuto.

In terzo luogo, se la semplificazione in sé è un intervento lodevole che porta a migliorare l'opera, lo è particolarmente alla luce del rapporto autore/pubblico. Gli autori illustri citati sopra nella loro sete di perfezionare le rispettive opere tenevano conto prima di tutto delle proprie esigenze. Severi nei 
propri confronti, ritenevano il pubblico altrettanto capace di giudicare l'alzamento di livello grazie alla semplificazione. Da questa prospettiva, la semplificazione non è un processo che miri ad abbassare il livello dell'opera:

(62) Che stiano dunque tranquilli i lettori, perché la loro competenza non sarà messa in discussione da un'edizione semplificata ed epurata dei termini e dei passaggi più ostici.

Anziché abbassare, la semplificazione permette di alzare ancora il livello dell'opera, raggiungendo il desiderato effetto della semplicità: l'armonia tra la forma e il contenuto, tra l'estetica e l'intelletto.

Fra i pochi commenti favorevoli alla semplificazione formulati dai lettori, la semplificazione è intesa piuttosto come «una facilitazione entro certi limiti»:

(63) Invece io la [=la versione riscritta] aspetto, favorevole alla riscrittura: sarà comunque arguta e allusiva. (Bisognerà mica ricordarlo che c'è semplificazione e semplificazione?)

Umberto Eco, nell'intervista concessa a Paolo Di Stefano, ovviamente adotta la concezione della semplificazione come un valore positivo. Anzi, come osserva malizioso, questa pratica, comune fra i letterati, lui l'aveva già applicata, solo che senza l'apposito annuncio non l'hanno notato neanche i critici della letteratura:

(64) Sono cose che fa chiunque. Sugli altri miei libri, di ristampa in ristampa, man mano che arrivavano segnalazioni di lettori e traduttori, cambiavo e correggevo, ma non se n'è accorto nessuno.

L'autore ammette di aver concesso poco a un pubblico culturalmente impreparato; anzi, la maggior parte delle modifiche le ha fatte in riferimento a un critico più esigente, ossia se stesso:

(65) In massima parte l'ho fatto per fastidio mio. Mi davano noia certe espressioni o ripetizioni. Per il lettore ho fatto piccoli aggiustamenti alleggerendo le citazioni latine, anche se avrei potuto fregarmene del lettore visto che il libro ha venduto trenta milioni di copie [...].

Nell'intervista, Umberto Eco inoltre critica le critiche incompetenti delle modifiche da lui introdotte:

(66) Ho visto un curioso articolo di Giuseppe Antonelli che diceva: Eco ha tolto settatore per mettere seguace e poi però due pagine dopo ha lasciato settatore... Ma è per quello che l'ho fatto, per evitare la ripetizione! È normale, se hai due volte la stessa parola non è che la sostituisci due volte.

Le modifiche effettivamente introdotte dall'autore non risultano limitarsi solo alla semplificazione, ma consistono pure in altri accorgimenti stilistici e contenutistici. 


\subsection{Competenza del tribunale (status translationis)}

Nel modello dello status quaestionis, l'ultima tappa della dinamica della disputa è lo status translationis. Nel contesto della disputa giuridica, la base del modello originale, se non era più possibile contestare né i fatti, né le definizioni, né le qualifiche, l'unica mossa rimasta per poter «vincere», pur momentaneamente, era contestare la competenza della corte che considerava la causa. Contestata la facoltà della corte a emettere il giudizio, si poteva argomentare che bisognava trasferire la causa a una corte competente, allontanando in questo modo il momento della pronuncia della sentenza che si temeva sfavorevole.

Nella disputa mediatica a proposito della versione semplificata de /l nome della rosa, le forme categorizzabili come manifestazioni dello status translationis sono le meno frequenti. Qualche internauta, stanco di leggere le infinite supposizioni riguardanti i fatti sconosciuti, contestava il «tribunale» sedicente, proponendo di trasferire il giudizio a un «tribunale» competente di chi, dopo la lettura della versione rinnovata, sarebbe stato capace di esprimersi in conoscenza di causa:

(67) Non sarebbe meglio prima leggere e poi commentare?

Anche Umberto Eco ha contestato la mancata competenza di chi si precipitava a giudicare senza prima appropriatamente informarsi:

(68) Ma sì, era un caso di scarsa serietà: si sono messi a parlare senza aver visto il libro [...].

Come si è visto sopra, proprio tali affermazioni - le idee senza il fondamento rigoroso nei fatti - sono proprio essenziali per rilevare le ideologie sottostanti.

\section{Conclusioni}

Il confronto tra le affermazioni formulate dai partecipanti alla disputa mediatica e quelle di Umberto Eco mostra che le modifiche, così come sono state descritte dall'autore, trascendevano l'ambito della sola semplificazione.

La disputa mediatica concerneva però proprio la semplificazione. Non conoscendo i fatti, i disputanti non potevano confrontarsi a livello dello status coniecturalis. Proprio per questo motivo, la polemica sull'imminente pubblicazione della versione modificata de /l nome della rosa si presta perfettamente alla ricostruzione delle ideologie della semplicità. Le controversie più accese, animate dall'adozione di diverse ideologie, riguardavano lo status qualitatis e, con meno intensità, lo status definitionis.

La discussione era così accesa per vari motivi. Riguardava un argomento rivelatosi palesemente importante ed interessante per i partecipanti. All'epoca la disputa non poteva essere risolta tramite il 
riferimento ai fatti. Inoltre, le argomentazioni e i giudizi non permettevano di arrivare a una soluzione, visto che scaturivano dalle incomprensioni concettuali di fondo. La fonte del conflitto era infatti la mancata distinzione fra i tipi di semplicità e i tipi di semplificazione. Siccome la semplificazione è il processo di raggiungimento della semplicità, quest'ultima è per forza presupposta in qualsiasi riferimento alla semplificazione.

Nella disputa sono apparse due accezioni qualitativamente diverse di semplicità. Per convenienza di esposizione esse saranno chiamate, rispettivamente, «la semplicità facile» e «la semplicità difficile». Gli corrispondono due nozioni di semplificazione: una degradante e condannabile, l'altra, intensificante e lodevole.

La semplicità facile è un pleonasmo che evoca l'immagine sottostante all'etimologia del termine semplice: «piegato una volta sola». Per eseguire il compito, ci si accontenta della prima costruzione che svolga la funzione comunicativa desiderata, non della migliore costruzione. Il testo è costruito in termini funzionali, non estetici. L'investimento dei mezzi intellettuali e delle risorse linguistiche nella produzione del testo sembra esiguo.

La semplicità difficile, a sua volta, è un ossimoro che si riferisce a un tipo qualitativamente diverso della semplicità. Ė una semplicità «concettuale». Una soluzione linguistico-testuale geniale nella sua semplicità si caratterizza per la sinteticità, l'eleganza, la parsimonia, l'economicità del messaggio e l'armonia, creando gli effetti di sinergia tra la forma e il contenuto. Essa costituisce un ideale, raramente raggiunto, della costruzione del messaggio.

Secondo la massima formulata all'inizio, «bisogna scrivere nella maniera più semplice possibile, ma non ancor più semplice». I critici della semplificazione de Il nome della rosa, volevano evitare che il testo fosse «ancor più semplice» - semplicistico o addirittura sempliciotto. In altre parole, protestavano contro il passaggio dalla semplicità difficile a quella facile, contro la primitivizzazione, marcata da perdite notevoli in termini di struttura, stile e spessore intellettuale. Da questa prospettiva, «meno è meno», e la semplificazione è degradante per il testo originale, dunque condannabile.

I fautori della semplificazione de II nome della rosa desideravano invece che il testo fosse «il più semplice possibile». Si riferivano dunque a una progressiva realizzazione della semplicità difficile, già parzialmente presente ne Il nome della rosa e sempre perfezionabile. Una tale semplificazione «migliorante» mira a intensificare gli effetti della semplicità difficile: la sinteticità, l'eleganza, la parsimonia, ecc. In questa prospettiva, «meno è più»: grazie alla sinteticità o all'eliminazione del superfluo si perfeziona il design del testo, ovvero la scorrevolezza, il ritmo, la musicalità, la plasticità, la densità del messaggio. Solo quando al testo non si può aggiungere nulla né togliergli nulla, è semplice - e perfetto. E non è semplice raggiungerlo. 


\title{
Bibliografia
}

\author{
Fonti primarie
}

$1^{\circ}$ gruppo

Assouline, Pierre (2011), «Eco réinvente son “Nom de la rose” pour les nuls», La république des livres. Le blogue de Pierre Assouline, disponibile su http://passouline.blog.lemonde.fr/2011/07/16/ eco-reinvente-son-nom-de-la-rose-pour-les-nuls/. [Sito consultato il 10 settembre 2013.]

Camillo, Andrea (2012), «"ll nome della rosa": mercoledì in libreria l'edizione "corretta e riveduta”», NewNotizie, disponibile su http://www.newnotizie.it/2012/01/il-nome-della-rosa-mercoledi-in-libreria-ledizione-corretta-e-riveduta/. [Sito consultato il 10 settembre 2013.]

Di Stefano, Paolo (2011), «Scrittori. L'arte di rifare. Correggere le proprie opere è l'ossessione dei grandi. Da Ariosto a Eco, il cantiere resta aperto», Corriere della sera, disponibile su http://www. corriere.it/cultura/11_settembre_02/di-stefano-arte-rifare_bef03b7e-d219-11e0-a205-8c1e98b416f7.shtml. [Sito consultato il 10 settembre 2013.]

Iovinella, Maria Rosaria (2011), «Eco senza spine. "Il nome della rosa” in versione semplice per i più giovani», Lettera 43, disponibile su http://www.lettera43.it/cultura/21899/eco-senza-spine. htm. [Sito consultato il 10 settembre 2013.]

Magi, Lucia (2011), «Umberto Eco prepara una nueva versión más “ágil” de "El nombre de la rosa”. El autor pretende hacer su obra "más accesible a los nuevos lectores" para aducuarla al siglo XX|», disponibile su http://cultura.elpais.com/cultura/2011/07/15/actualidad/1310680810_850215. html. [Sito consultato il 10 settembre 2013.]

Sorrentino, Luigia (2011a), «"I nome della rosa”, a settembre in vendita la nuova versione», Rai News, disponibile su http://poesia.blog.rainews.it/2011/08/19/il-nome-della-rosa-a-settembre-invendita-la-nuova-versione/\#sthash.uwToXLm2.dpuf. [Sito consultato il 10 settembre 2013.]

Sorrentino, Luigia (2011b), «Umberto Eco, “Boatos estivi”», Rai News, disponibile su http://poesia. blog.rainews.it/2011/09/05/umberto-eco-boatos-estivi/. [Sito consultato il 10 settembre 2013.]

Varasano, Lucia Grazia (2011), «"Il nome della rosa” dopo trent’anni riscritto e semplificato, Umberto Eco la riadatta ai giorni nostri», Mediapolitika, disponibile su http://www.mediapolitika.com/ wordpress/archivio/archives/14991. [Sito consultato il 10 settembre 2013.]

$2^{\circ}$ gruppo

http://capellidifata.it.forum-index.php?topic=1778.3705;wap2. [Sito consultato il 10 settembre 2013.] 
«Umberto Eco riscrive "Il nome della rosa". Sì, ok... ma perché?», Giramenti, disponibile su http:// gaialodovica.wordpress.com/2011/08/30/umberto-eco-riscrive-\%E2\%80\%9Cil-nome-dellarosa\%E2\%80\%9D-si-ok-ma-perche/. [Sito consultato il 10 settembre 2013.]

«Eco Riscrive II nome della rosa», Libri.tempoxme.it, disponibile su http://libri.tempoxme.it/index.php?option=com_content\&view=article\&id=437:eco-riscrive-il-nome-della-rosa\&catid=111:2011-08-agosto\&Itemid=6. [Sito consultato il 10 settembre 2013.]

«ll nome della rosa, la nuova edizone domani in libreria», Daring To Do, disponibile su http://www. daringtodo.com/lang/it/2012/01/09/il-nome-della-rosa-la-nuova-edizone-domani-in-libreria/. [Sito consultato il 10 settembre 2013.]

«Perché Eco ha riscritto "II nome della rosa”", Il Fatto Quotidiano, disponibile su http://www.ilfattoquotidiano.it/2011/08/11/perche-eco-ha-riscritto-il-nome-della-rosa/151044/. [Sito consultato il 10 settembre 2013.]

«Umberto Eco riscrive "Il nome della rosa"», Inchiostro \& Patatine. IL Forum Letterario, disponibile su http://www.ilforumletterario.com/t821-umberto-eco-riscrive-il-nome-della-rosa. [Sito consultato il 10 settembre 2013.]

«Questioni di stile», La Magia della Scrittura, disponibile su http://www.magiadellascrittura.it/?tag=linguaggio\&paged=2. [Sito consultato il 10 settembre 2013.]

http://www.pescepirata.it/aspiranti_scrittori/viewtopic.php?f=75\&t=1248. [Sito consultato il 10 settembre 2013.]

«Eco semplifica "Il Nome della Rosa" per le nuove generazioni», Spinoza, disponibile su http://www. spinoza.it/forum/viewtopic.php?f=3\&t=27600. [Sito consultato il 10 settembre 2013.]

«Se la montagna non va a Umberto Eco, Umberto Eco va alla montagna. Presto nelle librerie una versione semplificata de "Il nome della rosa"», TicinoLibero, disponibile su http://www.ticinolibero. ch/?p=77223. [Sito consultato il 10 settembre 2013.]

\section{Intervista con Umberto Eco}

Di Stefano, Paolo (2012), «Eco: così ho rivisto "Il nome della rosa" ma salvatemi dai critici militanti», Corriere della Sera, 31 gennaio 2012, http://www.libertaegiustizia.it/2012/01/31/eco-cosi-ho-rivisto-il-nome-della-rosa-ma-salvatemi-dai-critici-militanti/. [Sito consultato il 10 settembre 2013.]

\section{Riferimenti teorici}

Arystoteles (1996), Metafizyka, vol. I, Lublin, Redakcja Wydawnictw KUL.

Baldini, Massimo (1989), Parlar chiaro, parlare oscuro, Bari/Roma, Laterza.

Berthoz, Alain (2011), La semplessità, Torino, Codice Edizioni. 
Fiorentino, Giuliana (2009), «Complessità linguistica e variazione sintattica», Studi italiani di linguistica teorica ed applicata, vol. 38, n 2, p. 281-312.

Fioritto, Alfredo (1997), Manuale di stile. Strumenti per semplificare il linguaggio delle amministrazioni pubbliche, Bologna, Il Mulino.

Heath, Mark (1994), «The substructure of stasis-theory from Hermagoras to Hermogenes», Classical Quarterly, vol. 44, nº 1, p. 114-129.

Merrell, Floyd (2001), Simplicity and Complexity. Pondering Literature, Science, and Painting, Ann Arbor, The University of Michigan Press.

Patillon, Michel (1988), La théorie du discours chez Hermogène le rhéteur. Essai sur la structure de la rhétorique ancienne, Paris, Les Belles Lettres, coll «Études Anciennes ».

Piemontese, Maria Emanuela (1996), Capire e farsi capire. Teorie e tecniche della scrittura controllata, Napoli, Tecnodid.

Ponzio, Augusto (2006a), Linguaggio e relazioni sociali, Bari, Graphis.

Ponzio, Augusto (2006b), Produzione linguistica e ideologia sociale, Bari, Graphis.

Raboni, Giulia e Paola Italia (2011), Che cos'è la filologia d'autore, Roma, Carocci.

Tatarkiewicz, Władysław (1976), O doskonałości, Warszawa, PWN.

Van Dijk, Teun A. (1998), Ideology. A multidisciplinary approach, London, Sage.

Voghera, Miriam (1982), «La nozione di semplificazione come categoria interpretativa del parlato?», in Luciana Brasca e Maria Luisa Zambelli (ed.), Grammatica del parlare e dell'ascoltare a scuola, Quaderni del Giscel, n 13, Firenze, La Nuova Italia, p. 79-98.

Voghera, Miriam (2001), «Riflessioni su semplificazione, complessità e modalità di trasmissione: sintassi e semantica», in Maurizio Dardano, Adriana Pelo e Antonella Stefilongo (ed.), Scritto e parlato. Metodi, testi e contesti, Roma, Aracne, p. 65-78.

Załęska, Maria (2008), «ll troppo stroppia. Le critiche dell'expert writing nelle discipline umanistiche e sociali», in Ursula Reutner e Sabine Schwarze (ed.), Le style, c'est l'homme. Unité et pluralité du discours scientifique dans les langues romanes, Frankfurt am Main, Peter Lang, p. 205-226.

Załęska, Maria (2009), «Parodie dyskursu akademickiego», in Sylwia Dżereń-Głowacka e Alina Kwiatkowska (ed.), Humor. Teorie, praktyka, zastosowania / Humour. Theories, Applications, Practices. Vol. 1: Zrozumieć humor, Piotrków Trybunalski, Naukowe Wydawnictwo Piotrkowskie, p. 109-120. 